\title{
Morphology dependence of nanoparticle-on-mirror geometries: A quasinormal mode analysis
}

\author{
Kalun Bedingfield ${ }^{1, *}$, Eoin Elliott ${ }^{2}$, Nuttawut Kongsuwan ${ }^{3,4}$, Jeremy J. Baumberg ${ }^{2}$, and Angela Demetriadou ${ }^{1}$ \\ ${ }^{1}$ School of Physics and Astronomy, University of Birmingham, Edgbaston B15 2TT, UK \\ 2 NanoPhotonics Centre, Cavendish Laboratory, University of Cambridge, Cambridge CB3 0HE, UK \\ 3 The Blackett Laboratory, Prince Consort Road, Imperial College London, London SW7 2AZ, UK \\ ${ }^{4}$ Quantum Technology Foundation (Thailand), Bangkok 10110, Thailand
}

Received: 17 October 2021 / Accepted: 17 January 2022

\begin{abstract}
Plasmonic nanoantennas are able to produce extreme enhancements by concentrating electromagnetic fields into sub-wavelength volumes. Recently, one of the most commonly used nanoantennas is the nanoparticle-on-mirror geometry, which allowed for the room temperature strong coupling of a single molecule. Very few studies offer analysis of near-field mode decompositions, and they mainly focus on spherical and/or cylindrically-faceted nanoparticle-on-mirror geometries. Perfectly spherical nanoparticles are not easy to fabricate, with recent publications revealing that a rhombicuboctahedron is a commonly occurring nanoparticle shape-due to the crystalline nature of metallic nanoparticles. In this paper, we perform a quasi-normal mode analysis for the rhombicuboctahedron-on-mirror nanoantenna and map the field distributions of each mode. We examine how the geometry of the cavity defines the near-field distribution and energies of the modes, and we show that in some cases the mode degeneracies break. This has a significant impact on the radiative emission and far-field profile of each mode, which are measured experimentally. Understanding how realistic nanoantenna geometries behave in the near-field and far-field helps us design antennas with specific properties for controlling and sensing quantum emitters in plasmonic systems.
\end{abstract}

Keywords: polygon / NPoM / nanocavity / quasinormal mode / plasmonic nanoantenna

\section{Introduction}

Plasmonic nanoantennas are formed of two or more tightly coupled metallic nanostructures and-via the excitation of localised plasmons-are able to produce extreme field enhancements in the cavities that form between them. Thanks to recent advancements in nanofabrication techniques, plasmonic nanoantennas can now be realised with sub-nanometer cavity gaps-also known as nanocavities $[1,2]$. By concentrating electromagnetic (EM) fields into these sub-wavelength volumes, we form extreme nanoscale 'hot-spots' that enhance the incident light intensity by at least three orders of magnitude [3], which additionally offers efficient radiative far-field emission. Such an extraordinary environment has enabled many significant novel realisations, including: single molecule imaging [4], the interrogation of specific chemical bonds within a single molecule placed in a 'pico-cavity' [5], and even single molecule strong coupling at room temperature [6].

\footnotetext{
* e-mail: kjb896@bham.ac.uk
}

One of the simplest nanoantennas representative of this extreme plasmonic environment is that of a nanoparticle-on-mirror (NPoM) geometry; this is formed by assembling a nanoparticle (NP) on a flat substrate, forming a small gap between them. Literature has so far mainly concentrated on spherical (or truncated spherical) NPs-see Figure 1A. From both theoretical and experimental perspectives, the focus has predominantly been to analyse the optical response of plasmonic nanocavities via scattering methods. Very few papers offer analysis of near-field resonances or the decomposition of modes within the cavity [7-9], and instead often infer the resonant modes from far-field spectra. However, analysis of far-field results alone provides limited information on the non-radiative nature of the nearfield resonances and their interactions with quantum emitters (QEs) in the cavity, much less how radiation is emitted from the system [7].

It has been shown that although faceted spherical NPoM geometries offer a weaker field enhancement in the cavity-and therefore a smaller coupling strength to a single QE placed in the gap, due to the increased mode volume-it can support multiple QEs with an increased collective 

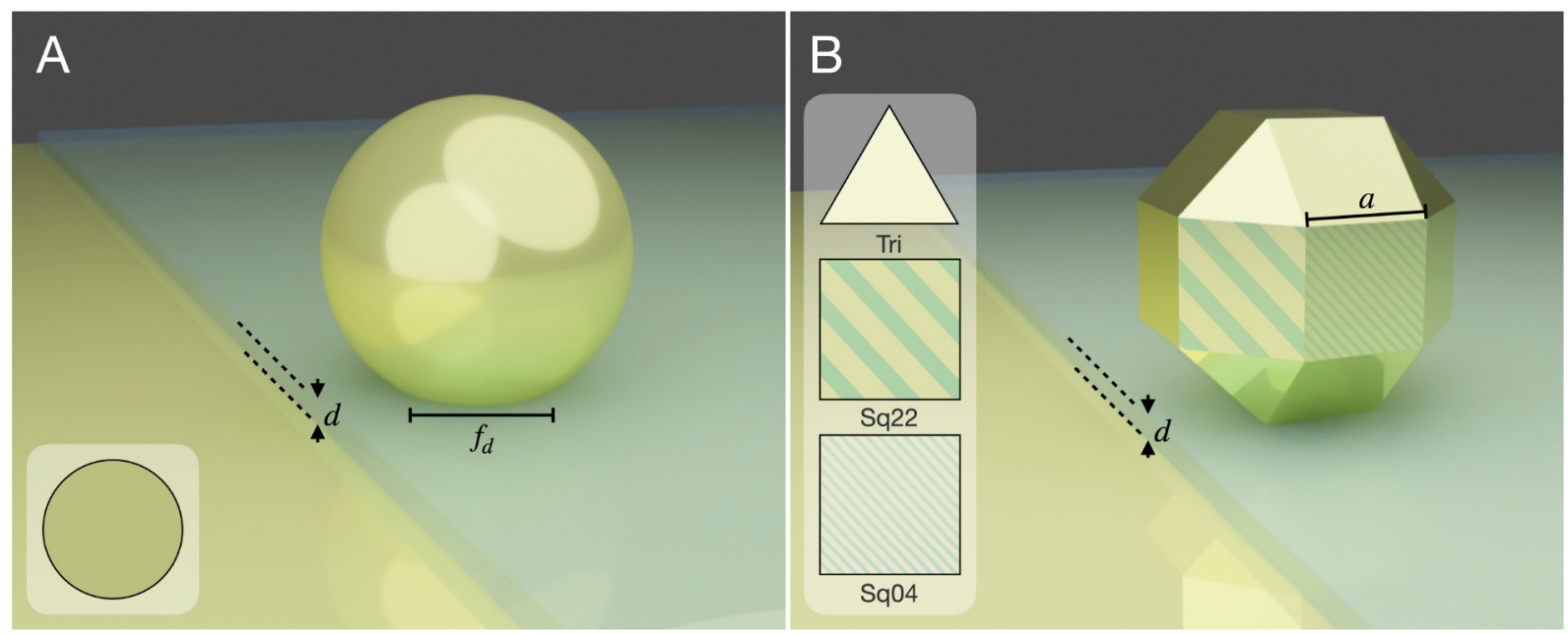

Fig. 1. Nanoparticle-on-mirror schematics for gold NPs assembled $d=1 \mathrm{~nm}$ above a gold substrate, separated by a spacer of refractive index $n=1.45$. A. Faceted spherical NPoM geometry for a $2 r_{p}=80 \mathrm{~nm}$ NP with a facet diameter of $f_{d}=20 \mathrm{~nm}$. The inset shows the circular facet that is in contact with the spacer. B. RhoM geometry for a NP with side length $a=30 \mathrm{~nm}$ and facet edge rounding $\rho=5 \mathrm{~nm}$. The inset shows the three unique facets that may be assembled on the substrate.

coupling strength, due to the coherent exchanging of energy [10]. The environment formed by this increased facet area is therefore ideal for the analysis of QEs in the strong coupling regime [6], and is also the perfect setting for the formation of pico-cavities, via the mobilisation of a single gold atom from the facet of the NP [5]. However, recent experimental work [11] has revealed that spherical NPs are actually polyhedrons, due to the crystalline nature of the metal. One of the most commonly occurring crystalline structures is the rhombicuboctahedron. Here we focus on the rhombicuboctahedron-on-mirror (RhoM) geometry-as shown in Figure 1B-which offers three unique facets that can be assembled on the substrate: two square and one triangular.

In this paper, we show using a quasi-normal mode (QNM) decomposition that the geometry of the facet (and therefore of the nanocavity formed) dictates: the near-field distribution of each mode, its resonant frequency, the radiative emission and far-field profile-as well as the energy degeneracies of the modes.

\section{Faceted spherical nanoparticle-on-mirror mode decomposition}

We start our discussion by exploring the near-field charge distributions of the decomposed QNMs within the cavity of a simple spherical faceted NPoM [7]. We also apply near-field to far-field transformation (NFFT) techniques to obtain the farfield Poynting flux from the sole knowledge of the near-fields in the vicinity of the cavity. For complicated geometries such as this, analytic modal decompositions are not generally available and we must turn to numerical methods instead to describe the modes of open and dissipative systems, forming a set of complex eigenfrequencies [8]. For dispersive media such as gold, we employ the efficient QNM solver: QNMEig, which follows an auxiliary-field eigenvalue approach [9,12]. The symmetry of this system allows the spherical harmonic nomenclature to be adopted to describe the decomposed near-field charge distributions-see Kongsuwan et al. [7] -where each QNM is labelled by $i=(l, m)$, for a set of positive integers $l$ and $-l \leq m \leq l$.

The first eight near-field QNMs of the faceted spherical NPoM are shown in Figure 2A. These QNMs reveal a series of complex charge distribution patterns, and the field enhancement is very clearly confined to the extents of the facet. Modes of $m=0$ display central maxima, whereas modes of $m=l$ exhibit $2 m$-lobed charge distributions, as expected. Higher order $m \neq l$ modes have the same behaviour as their $m=l$ counterparts with an additional pair of oscillations along the $2 m$-lobes, concentrically compressing the mode profile within the facet.

To highlight the energetic ordering of the modes, Figure 3 fits the complex QNM eigenfrequencies to Lorentzians for the same eight modes. Due to the cylindrical symmetry of the facet, all $(l,|m|)$ mode pairs are degenerate and oriented orthogonal to each other. In comparison to the mode ordering of the spherical NPoM shown in Kongsuwan et al. [7], the introduction of the facet significantly alters the energetic favourability of the modes-depending on the size of the facet in comparison to the spatial arrangement of the charge distributions.

From these near-field QNMs profiles, the respective farfield emission profiles can be obtained via a NFFT. Here we use RETOP $[13,14]$, which solely considers the QNM nearfields (shown in Fig. 2A) on the surface of a box surrounding the NP and cavity. Then, using RETOP, the structure is illuminated with plane waves, and by applying the Lorentz reciprocity theorem, RETOP calculates the radiating fields (specifically the power flux) emitted from the system to the far-field. The time averaged Poynting flux reaching the farfield for the same eight QNMs of the faceted NPoM are shown in Figure 2B. These are plotted in polar coordinates, with the polar angle $(\theta)$ increasing from the centre. Modes of $m=0$ reveal a ring-like emission pattern, with a peak angle of emission at $\theta=62^{\circ}$-whereas the $(1,1)$ and $(2,2)$ modes retain their $2 m$-lobed nature. 
A
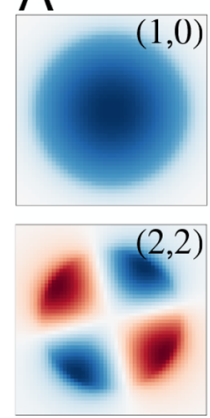

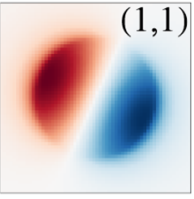

$(2,0)$
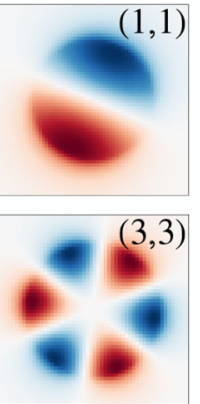
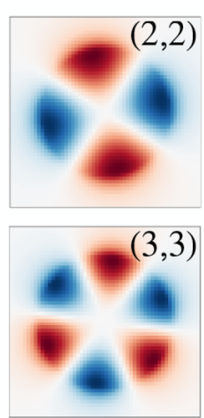
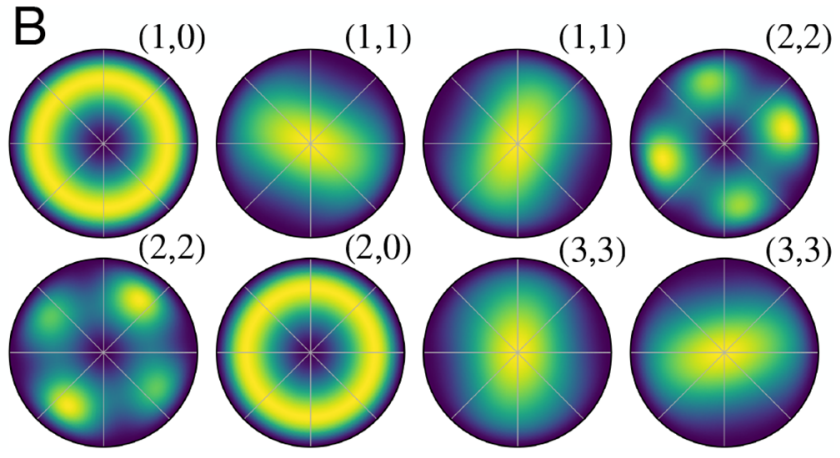

Fig. 2. QNMs of the circularly faceted spherical NPoM geometry, ordered in increasing energy: $(1,0),(1,1),(1,1),(2,2),(2,2),(2,0)$, $(3,3),(3,3)$. A. Normalised QNM electric fields $E_{z, l m}$ in the $x y$-plane through the centre of the cavity. B. Time averaged far-field Poynting flux $\left\langle S_{l m}\right\rangle$.

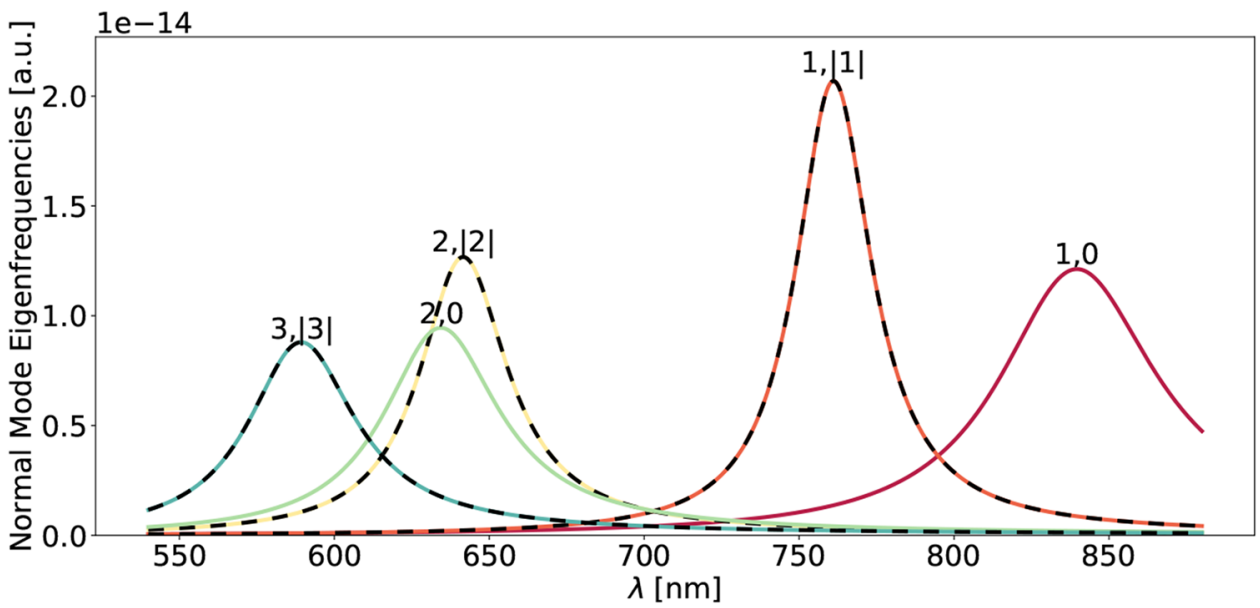

Fig. 3. Faceted spherical NPoM QNM complex eigenfrequencies fitted to Lorentzians. Dashed lines represent degenerate QNMs.

\section{Rhombicuboctahedron-on-mirror mode decomposition}

While the faceted spherical NPoM gives us a good understanding and nomenclature of the supported modes and the radiative emission profiles, the structure's geometry is very idealized. A more realistic NP geometry is the rhombicuboctahedron NP, which is formed due to the crystalline nature of gold [11]. The rhombicuboctahedron can be assembled on a mirror to form a rhombicuboctahedron-on-mirror (RhoM). This has multiple distinct facet orientations that can be assembled on the substrate to form different nanocavity geometries, each supporting a separate set of QNMs with very interesting properties: two square facets-one with four square neighbours (Sq04), and one with two square and two triangular neighbours (Sq22)-and a triangular facet, with four square neighbours. The three different facets are highlighted in the inset of Figure 1B. The morphology of the cavity is significantly altered depending on which facet of the NP is assembled on the substrate. We focus on the two square facets, exploring the effects of the differing neighbouring sides on both the near-field and far-field profiles.
Employing the same QNM solver as used for the spherical faceted NPoM system above, we determine the set of QNM profiles and corresponding complex eigenfrequencies for the Sq04 RhoM-as shown in Figure 4A. Although the structure is no longer cylindrically symmetric the spherical harmonic nomenclature still applies to the modes of the RhoM facets. It is clear that this square facet supports $m=0$ modes with a central maxima, and modes of $m=l$ exhibit $2 m$-lobed charge distributions similar to the spherical faceted NPoM. In contrast to the modes of the faceted NPoM-where the symmetry allows the modes to appear at any azimuthal angle, as long as mode pairs are orthogonal-the modes of the Sq04 RhoM preferentially reorientate such that the field extrema align with the corners of the facet. Not only does this significantly alter the spatial arrangement of the charges, but the differing ability of each mode to align with the facet corners leads to an energetic reordering of the modes-this is highlighted in the spectra of Figure 5A. More specifically, even- $m$ mode pairs have one mode which preferentially aligns with the facet corners, and one mode that must be orientated perpendicularly and therefore have its extrema align with the facet sides. The latter of these is much less energetically favourable, and therefore leads to a splitting of even- $m$ mode pairs-such as the $(2,2)$ modes. 

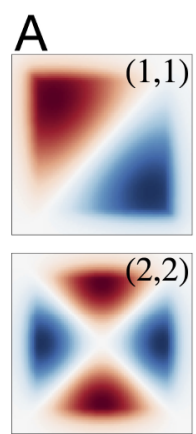

C
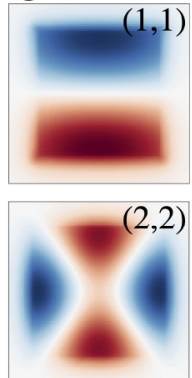

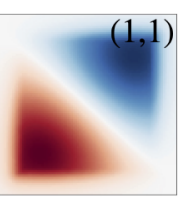

$(2,0)$
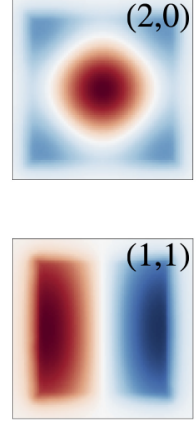

$(2,0)$

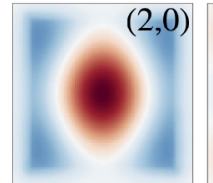

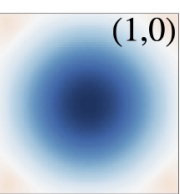
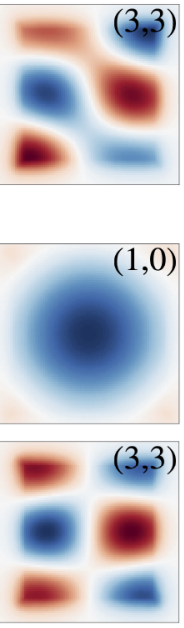
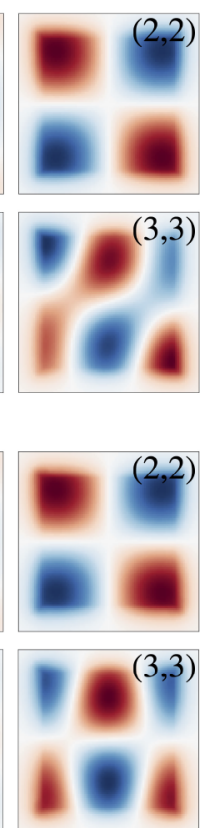

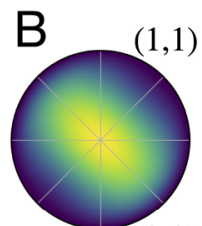

$(2,2)$
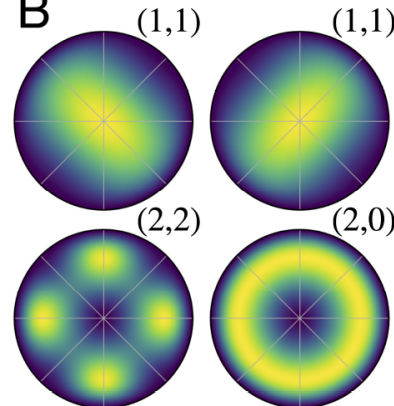

$(2,0)$
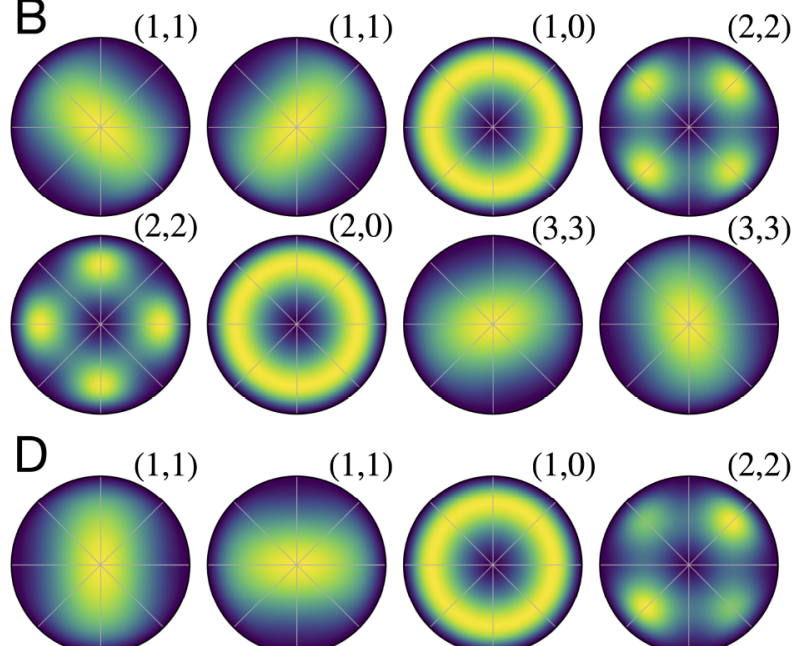

$(2,2)$
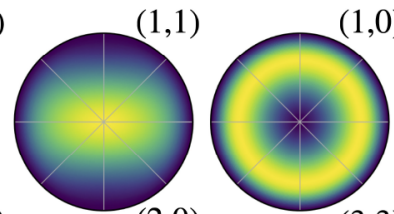

$(1,0)$

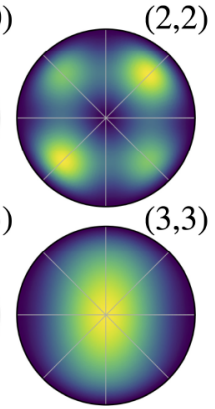

Fig. 4. QNMs of the RhoM geometry assembled on the (A,B) Sq04 and (C,D) Sq22 facets, ordered in increasing energy: (1,1), (1,1), $(1,0),(2,2),(2,2),(2,0),(3,3),(3,3)$. A,C. Normalised QNM electric fields $E_{z, l m}$ in the $x y$-plane through the centre of the cavity. B,D. Time averaged far-field Poynting flux $\left\langle S_{l m}\right\rangle$.

Again using RETOP, the far-field Poynting flux is found for each QNM and is shown in Figure 4B. Similarly to the faceted NPoM, the far-field profiles for the Sq04 RhoM emit with a ring-shaped emission pattern for $m=0$ modes-at the same peak emission angle-with the $(1,1)$ and $(2,2)$ modes also retaining their $2 m$-lobed nature. The emission of higher order modes, such as the $(3,3)$ modes, are instead dominated by the dipoles that form between the six-lobes of the near-field distribution-hence the reason these modes are mostly orientated across the sides of the facet, but are slightly angled by the enhanced lobes in the facet corners.

Unlike the four square neighbours of the $\mathrm{Sq} 04$ facet, the triangular neighbours of the $\mathrm{Sq} 22$ facet reside at an angle closer to the substrate, and therefore lead to a greater field enhancement on the neighbouring sides of Sq22 facet. This forms an unequal confinement across the Sq22 facet, effectively elongating it. This effect dominates over the increased field enhancement in the facet corners for the $m=1$ modes, causing them to reorientate and align with the sides of the facet-as shown in Figure 4C-rather than the corners, as the Sq04 did. Relative to the QNM profiles shown here, the triangular facets neighbour at the top and bottom-evidenced by the energetic favourability of the $(1,1)$ mode that aligns along this direction. With the additional symmetry broken by the unequal confinement across the facet, its supported modes receive the additional energetic splitting of odd- $m$ modes-as shown in Figure 5B. This demonstrates the larger splitting observed for even- $m$ modes due to the perpendicular orientation between more pairs in a square facet, and the smaller splitting of odd- $m$ mode pairs from the effectively elongated facet and the unequal confinement this produces.

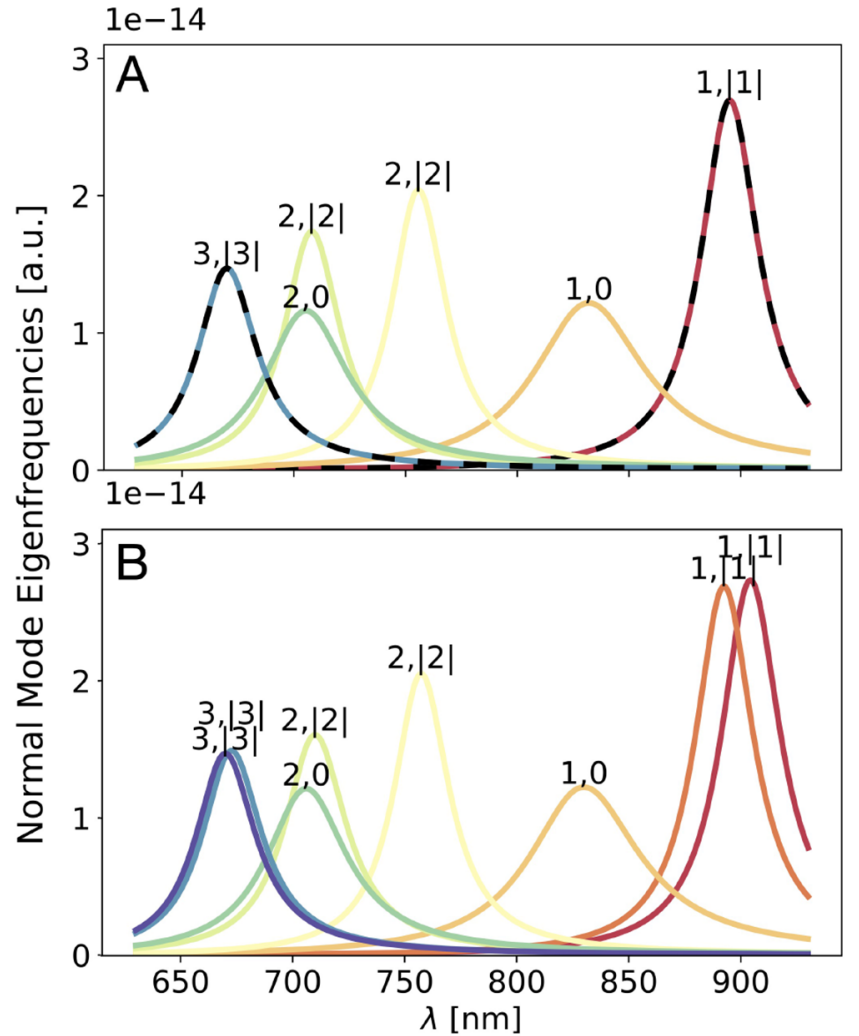

Fig. 5. RhoM QNM complex eigenfrequencies fitted to Lorentzians for the (A) Sq04 and (B) Sq22 facet orientations. Dashed lines represent degenerate QNMs. 
For the majority of modes, the far-field profiles are largely unchanged-see Figure 4D. The exception being non-zero even- $m$ modes, where one of the modes of this pair aligns its same-sign extrema with the effective elongation axis-for example, the second $(2,2)$ mode. This stretches the mode such that the central node is lost. This has a profound effect such that the far-field is dominated by the non-zero central field-resulting in an emission profile that resembles an $m=0$ mode in spite of the $2 m$-lobed near-field charge distribution. The understanding we have gained on the supported QNMs, energetic arrangements and radiative emissions of these distinct nanoantenna geometries is essential for the analysis of experimental far-field results-as well as for tailoring the design of plasmonic nanoantennas to offer specific near-field enhancements for the placement of QEs.

\section{Conclusion}

In the past few years, particles assembled on a flat metal surface have provided a pathway to form plasmonic nanocavities with extreme light confinement, which has led to significant experimental breakthroughs. Theoretical descriptions of such nanocavities has so far mainly concentrated on spherical and/or truncated particles. However, NPs are polyhedrons due to the crystalline nature of metal, with a rhombicuboctahedron being one of the most commonly occurring shapes. Here we adopt a quasi-normal mode approach to decompose the modes of a nanocavity formed by a rhombicuboctahedron-on-amirror (RhoM). We find that the different facets of the rhombicuboctahedron NP create different nanocavities, with very distinct near-field distributions, energies, radiative emissions and far-field profiles. More specifically, the symmetry of the facet and its neighbouring faces dictates the near-field modes and their degeneracies, with the far-field emissions following closely the near-field changes. By understanding the modal response of realistic nanoantenna geometries in both the near-field and farfield aids in the designing of antennas with specific features for sensing and interacting with QEs in plasmonic systems.
$\mathrm{AD}$ gratefully acknowledges support from the Royal Society University Research Fellowship URF $\backslash$ R1 180097, Royal Society Research Fellows Enhancement Award RGF $\backslash$ EA $\backslash 181038$, Royal Society Research grants RGS $\backslash R 1 \backslash 211093$ and funding from EPSRC for the CDT in Topological Design EP/S02297X/1.

\section{References}

1. F. Benz, C. Tserkezis, L.O. Herrmann, B. De Nijs, A. Sanders, D.O. Sigle, L. Pukenas, S.D. Evans, J. Aizpurua, J.J. Baumberg, Nano Lett. 15, 669 (2015)

2. A. Emboras, J. Niegemann, P. Ma, C. Haffner, A. Pedersen, M. Luisier, C. Hafner, T. Schimmel, J. Leuthold, Nano Lett. 16, 709 (2016)

3. J.J. Baumberg, J. Aizpurua, M.H. Mikkelsen, D.R. Smith, Nat. Mater. 18, 668 (2019)

4. B. Yang, G. Chen, A. Ghafoor, Y. Zhang, Y. Zhang, Y. Zhang, Y. Luo, J. Yang, V. Sandoghdar, J. Aizpurua, Z. Dong, J.G. Hou, Nat. Photonics 14, 693 (2020)

5. F. Benz, M.K. Schmidt, A. Dreismann, R. Chikkaraddy, Y. Zhang, A. Demetriadou, C. Carnegie, H. Ohadi, B. De Nijs, R. Esteban, J. Aizpurua, J.J. Baumberg, Science 354, 726 (2016)

6. R. Chikkaraddy, B. De Nijs, F. Benz, S.J. Barrow, O.A. Scherman, E. Rosta, A. Demetriadou, P. Fox, O. Hess, J.J. Baumberg, Nature 535, 127 (2016)

7. N. Kongsuwan, A. Demetriadou, M. Horton, R. Chikkaraddy, J.J. Baumberg, O. Hess, ACS Photonics 7, 463 (2020)

8. P.T. Kristensen, R.C. Ge, S. Hughes, Phys. Rev. A 92, $053810(2015)$

9. C. Sauvan, J.P. Hugonin, I.S. Maksymov, P. Lalanne, Phys. Rev. Lett. 110, 237401 (2013)

10. N. Kongsuwan, A. Demetriadou, R. Chikkaraddy, J.J. Baumberg, O. Ortwin, EPJ Appl. Metamat. 5, 6 (2018)

11. F. Benz, R. Chikkaraddy, A. Salmon, H. Ohadi, B. De Nijs, J. Mertens, C. Carnegie, R.W. Bowman, J.J. Baumberg, J. Phys. Chem. Lett. 7, 2264 (2016)

12. P. Lalanne, Light-in-complex-nanostructures/MAN: Versions 7.1 of QNMEig and QNMPole, Zenodo (2020)

13. J. Yang, J.P. Hugonin, P. Lalanne, Light-in-complexnanostructures/RETOP: Version 8.1, Zenodo (2020)

14. J. Yang, J.P. Hugonin, ACS Photonics 3, 395 (2016)

Cite this article as: Kalun Bedingfield, Eoin Elliott, Nuttawut Kongsuwan, Jeremy J. Baumberg, Angela Demetriadou, Morphology dependence of nanoparticle-on-mirror geometries: A quasinormal mode analysis, EPJ Appl. Metamat. 9, 3 (2022) 